\title{
Mammary Gland Malignant Myoepithelioma in a Domestic Rabbit (Oryctolagus cuniculus)
}

\author{
Halana do Carmo Silva', Ayisa Rodrigues de Oliveira', Rodrigo dos Santos Horta', Betânia Souza Monteiro', \\ Tatiany Luiza Silveira ${ }^{3}$, Giovanni Dantas Cassali ${ }^{3} \&$ Mayra Cunha Flecher ${ }^{1}$
}

\begin{abstract}
Background: Malignant myoepithelioma is a rare neoplasm in humans and rats, poorly reported in dogs and not previously described in rabbits. This study aims to report a case of malignant mammary myoepithelioma in a domestic rabbit (Oryctolagus cuniculus).

Case: A domestic, hybrid, female, entire, nulliparous, 7-year-old rabbit was attended in the Veterinary Hospital Prof. Ricardo Alexandre Hippler at Universidade Vila Velha (UVV) with a history of apathy, lethargy and anorexia. In the clinical examination a $5 \mathrm{~cm}$ tumour was observed in the right abdominal mammary gland (M2), along with floating smaller lesions in M1 and M3. There were no palpable abnormalities in the axillary and inguinal lymph nodes. Complete blood count and serum biochemistry were within the normal range. Fine needle aspiration was performed for cytological evaluation of the tumour in M2 and it was suggestive of a malignant glandular neoplasm, with probable epithelial origin. Complete staging included chest and abdominal radiographs, but they were unremarkable. The patient was submitted to a right unilateral mastectomy, involving resection of the inguinal lymph node. The sample was sent to the Laboratory of Animal Pathology of UVV for routine histological evaluation. M2 lesion was compatible with malignant myoepithelioma based on the literature, while nodules in M1 and M3 were classified as mammary cysts. The inguinal lymph node showed no significant abnormalities. An immunohistochemical panel was performed in the Laboratory of Comparative Pathology of the Institute of Biological Sciences at the Universidade Federal de Minas Gerais (UFMG), using immunomarkers (pan-cytokeratin, p63 and vimentin) to characterize the tumour and confirm its diagnosis. Immunohistochemistry revealed expression of p63, and was negative for pan-cytokeratin and vimentin, which confirmed the myoepithelial origin, without involvement of the epithelial compartment. Clinical follow-up was recommended every 2-3 months, including abdominal ultrasonography, chest radiographs, complete blood count and serum biochemistry. Ultrasonography showed thinning of the uterine wall, dilation of $3.7 \mathrm{~cm}$ in diameter and presence of endometrial cysts of varied sizes, around $0.7 \mathrm{~cm}$. Neutering was recommended but it was not elected by the pet's responsible. These findings remained stable throughout the follow-up, which was greater than 405 days.

Discussion: This is the first report of a malignant myoepithelioma in rabbit, with a detailed description of the clinical, surgical, pathological and immunophenotypic aspects of this neoplasm in this species. This is a tumour characterized by proliferation of epithelioid to spindle-like myoepithelial cells, as observed in this case, forming an extensive and well delimited solid area with marked central necrosis, compatible with what has already been described in the literature in bitches and women. As observed in this case, the neoplastic cells usually exhibit moderate cellular and nuclear pleomorphism, moderate anisocytosis and anisokaryosis, and several mitotic figures; different from what is observed in women, in which mitotic figures vary from small to moderate amount. Neoplasia and mammary cysts were removed by mastectomy, and the owner choose not to spay the animal. On ultrasonography, after 12 months, the endometrium presented structures similar to cysts and it was hyperplastic, which is commonly reported in association to mammary neoplasia.
\end{abstract}

Keywords: oncology, exotic animal, mammary gland, lagomorph, pathology.

DOI: $10.22456 / 1679-9216.92568$ 


\section{INTRODUCTION}

Neoplasms of the mammary gland are relatively frequent in rabbits, often concomitantly with uterine hyperplasia or adenocarcinomas, suggesting a relationship between these conditions [26]. Although the biological behaviour is poorly characterized and metastatic potential remains unknown, at least $70 \%$ of the mammary tumours described in rabbits are malignant and may develop metastases to regional lymph nodes, lungs, liver, kidneys, pancreas, adrenal glands, ovaries, and bone marrow [20]. The most commonly reported histological types are invasive carcinoma [20] and tubular carcinoma [3].

Malignant myoepithelioma is a rare neoplasm in humans [15,24] and in rats [2], poorly reported in dogs [17] and not previously described in rabbits. The origin of this tumour is the myoepithelial cell, which presents a fusiform to starry appearance, involving the epithelial cells of the ducts and breast alveoli $[4,8]$. Confirmation of this diagnosis requires the use of immunohistochemical markers to confirm the presence of myoepithelial neoplastic cells and differentiate them from the other cellular components of the mammary gland $[4,7,8]$.

Neoplastic cells may be epithelioid, with nests, to fusiform (myoepithelium), and may contain myxoid and pleomorphic matrix $[8,24]$. The benign form of this tumour is named myoepithelioma, but it may be named adenomyoepithelioma or complex adenoma when there is associated epithelial (tubular) proliferation with cartilaginous or bone differentiation [8]. Malignant myoepithelioma has been also reported in other glands such as the salivary gland of a dog [6] but has not been reported yet in rabbits.

The aim of this study is to report a case of malignant myoepithelioma of the mammary gland in a female rabbit, to document its clinical, cytological, histological and immunohistochemical features, and to improve the knowledge about its biological behaviour.

\section{CASE}

A hybrid domestic rabbit (Oryctolagus cuniculus), female, entire, nulliparous, with 7-year-old and $3.3 \mathrm{~kg}$ was attended in the Veterinary Hospital Prof. Ricardo Alexandre Hippler at Universidade Vila Velha (UVV) with a history of apathy, lethargy and anorexia. In the clinical examination, a $5 \mathrm{~cm}$ tumour was observed in the right abdominal mammary gland (M2), without ulcerations or adherence to the skin or musculature, along with floating smaller lesions in M1 and M3. No palpable macroscopic abnormalities were observed in the axillary and inguinal lymph nodes. The complete blood count and serum biochemistry (urea, creatinine, alkaline phosphatase and alanine aminotransferase) were within normal ranges.

Fine needle aspiration was performed for cytological evaluation of the tumour. Microscopic evaluation revealed many epithelioid to fusiform cells with moderate adhesiveness, organized into groups and sometimes individualized. The cells had bluish cytoplasm, containing mild to moderate amount of intracytoplasmic vacuoles. There was an increase in the nucleus: cytoplasm ratio. The nuclei were large, round, with coarse chromatin and a single evident nucleolus; presenting moderate anisokaryosis and cellular pleomorphism. Two mitotic figures were observed (400x). Among the cells there was a moderate amount of amorphous pink material. Cytological findings were suggestive of malignant glandular neoplasia, with probable epithelial origin (Figure 1A).

Because of the evidence of malignant neoplasm, a complete staging was carried out with thoracic and abdominal radiographs in three projections (left and right lateral and ventrodorsal), but they were unremarkable. Right radical mastectomy was recommended along with inguinal lymphadenectomy. The patient was premedicated with $1 \mathrm{mg} \mathrm{kg}^{-1}$ intramuscular of acepromazine $\left(\text { Acepran }^{\circledR}\right)^{1}$. After $20 \mathrm{~min}$, wide trichotomy of the thoracic and abdominal region was performed, and the lateral vein of the ear was catheterized. Anesthetic induction was made using the association of 30 $\mathrm{mg} \mathrm{kg}^{-1}$ and $0.5 \mathrm{mg} \mathrm{kg}^{-1}$ intravenous, respectively, of ketamine $\left(\text { Cetamin }^{\circledR}\right)^{2}$ and midazolam $\left(\text { Midazolam }{ }^{\circledR}\right)^{3}$, followed by orotracheal intubation and anesthetic maintenance with isoflurane (Isoflurano $\left.{ }^{\circledR}\right)^{4}$ of 1,5-2-\% minimum alveolar concentration (MAC) diluted in medicinal oxygen, in an open system (Rees-Baraka). The patient was placed in dorsal recumbency and the antisepsis was performed with chlorhexidine $2 \%$ and alcohol $70 \%$. An elliptic skin incision was carried out involving all the mammary glands of the right chain, followed by dissection of the subcutaneous tissue and the mammary glands to the muscular fascia. The artery and superficial epigastric vein were localized and sutured with synthetic absorbable yarn (poliglactina acid, 2-0) and sectioned for resection of the breast tissue, along with the inguinal lymph node. Suture of 


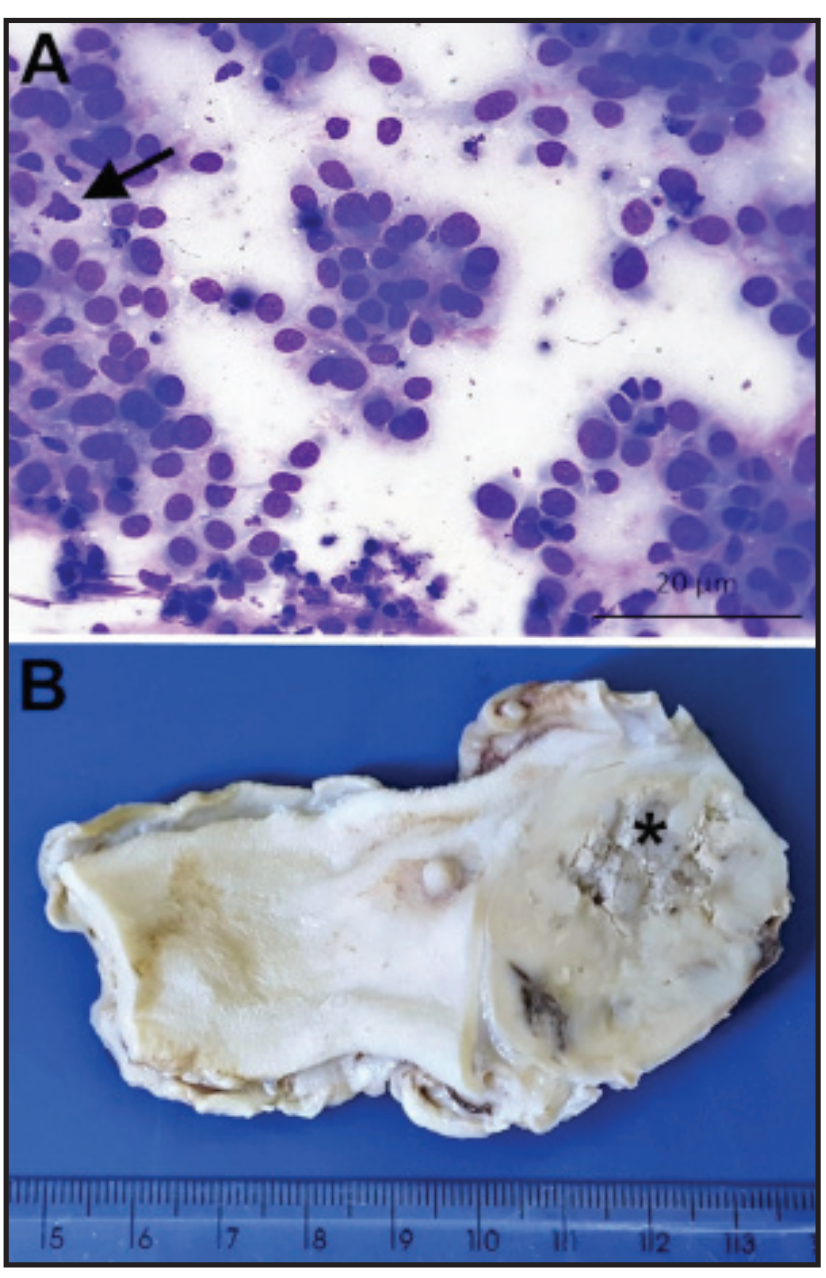

Figure 1. A- Mammary malignant myoepithelioma in a domestic rabbit. Fine needle aspiration cytology of a mammary gland mass.. Field with large amounts of oval or stellate cells with moderate adhesiveness and high nuclei: cytoplasm ratio. Presence of amorphous eosinophilic intercellular material and mitotic figures (arrow) [Diff-Quick, 400x]. B- Mammary malignant myoepithelioma in domestic rabbit. Macroscopy of the right abdominal mammary gland presenting tumour with $4,0 \mathrm{~cm}$ x 3,5 cm x 3,5 $\mathrm{cm}$ and circular contour well-delimited and non-ulcerating. Whitish cutting surface with blackened, multifocal friable tissue with necrotic aspect $\left(^{*}\right)$.

subcutaneous tissue with Sultan pattern was performed using synthetic absorbable yarn (poliglactina acid, 2-0), and dermorrhaphy with Wolff pattern and synthetic non-absorbable yarn (nylon, 3-0).

The resected tissues were fixed in $10 \%$ buffered formalin and sent for routine histological processing in the Laboratory of Animal Pathology at UVV and stained with hematoxylin and eosin (HE). Macroscopically, the right abdominal mammary gland (M2) presented a circular, soft volume, slightly adhered to the musculature, non-ulcerated and had a yellowish-white surface with dark gray and friable areas (necrosis) (Figure 1B). The adjacent mammary glands (M1 and M3, thoracic and inguinal, respectively) had small cystic structures of varying sizes flowing whitish milky material to the cut. A single inguinal lymph node was identified, measuring $0.1 \mathrm{~mm}$ in diameter, without macroscopic abnormalities.

Microscopically, a solid, multilobulated, well delimited, unencapsulated proliferation was observed in the abdominal mammary gland extending to the deep dermis. The lobules were separated by a thin septum of fibrovascular connective tissue (Figure 2A\&B). Neoplastic cells were spindle-like to epithelioid and had abundant eosinophilic cytoplasm, with intracytoplasmic vacuoles. It was observed a high nucleus: cytoplasm ratio. The nuclei were large, round to oval, with occasional and evident double to multiples nucleoli. There was moderate karyomegaly, marked anisokaryosis, marked cellular pleomorphism, and moderate nuclear pleomorphism. A total of 47 mitosis figures were observed in 10 high power field (400x). Sometimes the cells acquired a spiral pattern forming small swirls (Figure 2C). In the most central regions of the neoplasia, large areas of necrosis and hemorrhage were observed. There was also marked desmoplasia and moderate amount of amorphous amphophilic material (mucin) adjacent to the neoformation. The histological characteristics were compatible with mammary malignant myoepithelioma, based on the classification of Cassali et al. [4] and Goldschmidt et al. [8] for canine's mammary neoplasms. In the thoracic and inguinal mammary glands intense ductal ectasia was observed with large amount of eosinophilic material in the interior and flattening of the epithelium of the lining. These were classified as mammary cysts, according to Schöniger et al. [20]. The inguinal lymph node showed no significant microscopic abnormalities.

For the characterization of the neoplasm and confirmation of the diagnosis of malignant myoepithelioma an immunohistochemical panel was carried out in the Laboratory of Comparative Pathology (LPC) of the Institute of Biological Sciences at Universidade Federal de Minas Gerais (UFMG), using the immunomarkers described in Table 1. Immunohistochemistry was performed in accordance with the standardized protocol by LPC. The tumour presented positive immunolabeling for p63 (Figure 2D) and negative for the remaining markers in the neoplastic cells. In addition to the internal controls, it was used as a positive control of the reactions a sample of canine mammary carcinoma and as a negative control the same tumour, with suppression of primary antibody. 


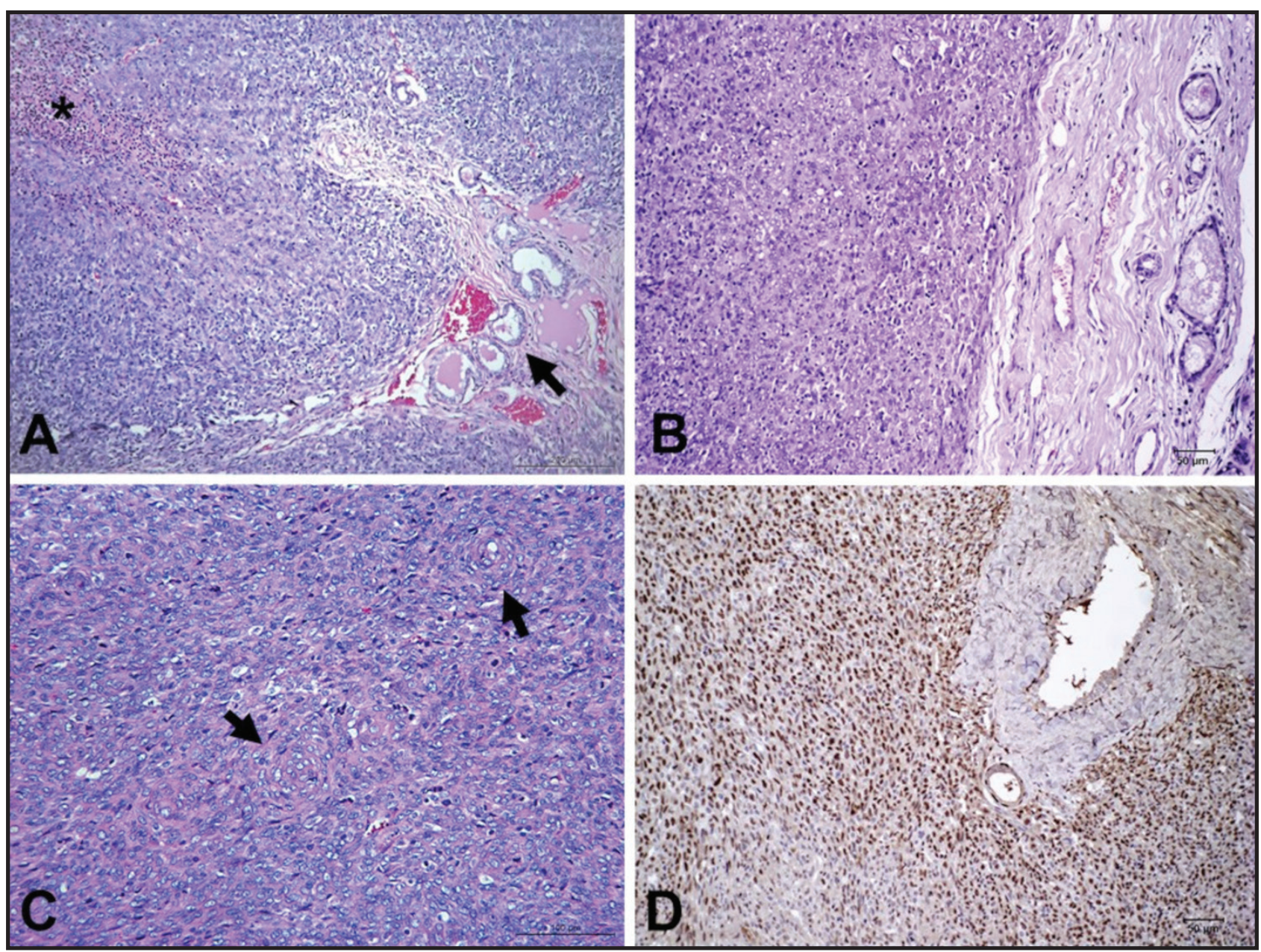

Figure 2. A- Mammary gland. Solid neoformation with central area of necrosis (*). On the arrow, breast lobes adjacent to the neoformation with no abnormalities [HE, 100x]. B- Mammary gland. Well delimited solid neoformation [HE, 100x]. C- Mammary gland. Neoformation with neoplastic cells with high nuclei: cytoplasm ratio; large nuclei, round to ovals, vesicular with single to double evident nucleolus. High cellular pleomorphism and moderate nuclear pleomorphism. Sometimes cells acquired a spiral pattern (arrows) [HE, 400x]. D- Mammary gland. Immuno-immunohistochemistry with positive immunolabeling for p63 [100x].

Table 1. Sources, clones and dilutions of primary antibodies.

\begin{tabular}{cccc}
\hline Antibody & Brand & Clone & Dilution \\
\hline Pan-ctytokeratin $^{5}$ & Dako & AE1/AE3 & $1: 500$ \\
Vimentin $^{5}$ & Dako & V9 & $1: 800$ \\
p63 $^{6}$ & Neomarks & 4A4 & $1: 100$ \\
\hline
\end{tabular}

The patient recovered well after surgery and the external sutures were removed after 10 days. Considering the malignant tumour and the absence of well-established chemotherapeutic protocols for mammary tumours in rabbits, clinical follow-up was recommended every 2-3 months, including abdominal ultrasonography, chest radiographs (three projections - right and left lateral, ventrodorsal), complete blood count and serum biochemistry. No abnormalities were found in the hematological examinations and radiographs, however, ultrasound showed thinning of the uterine wall, dilation of $3.7 \mathrm{~cm}$ in diameter and presence of endometrial cysts of varied size, around $0.7 \mathrm{~cm}$. Neutering was recommended but it was not elected by the pet's responsible. These findings remained stable throughout the follow-up, which was greater than 405 days.

\section{DISCUSSION}

This is the first report of a malignant myoepithelioma in a rabbit, with detailed description of the clinical, surgical, pathological and immunophenotypical features of this neoplasm in this species. It is a 
tumour characterized by the proliferation of epithelioid to fusiform myoepithelial cells $[4,8]$, as observed in this case, forming an extensive and well delimitated solid area with sharp central necrosis, compatible with what has already been described in the literature in bitches [4] and women [14,24]. The cells, as noted in this case, usually display moderate cellular and nuclear pleomorphism, moderate anisocytosis and anisokaryosis, and numerous mitotic figures [8]. All these features were found in the described case, including necrosis and the high quantity of mitotic figures. In women, reported cases are presented with fewer mitotic figures, which varies from a few to moderate [14,24], different from this report in which the mitotic activity was high.

Immunohistochemistry is required for confirmation of the diagnosis of malignant myoepithelioma, using the markers for epithelial cells such as cytokeratin (AE1/AE3, CK14, CK8/18), myoepithelial (smooth muscle actin-SMA, calponin, p63) and mesenchymal cells (vimentin) $[8,13,23]$. The p63 marker, a counterpart of the p53, is a marker of the myoepithelial cells, expressed only in the nucleus, in normal and neoplastic mammary glands. It is highly sensitive and specific to myoepithelial cells in mammary neoplasms, also used in rabbits [20], when compared to other markers for the same cellular type [7]. The tumour identified in this case presented a high expression of $\mathrm{p} 63$, which proved to be a neoplasm of myoepithelial origin, without compromising the epithelial compartment, since there was no immunolabeling for AE1/AE3 in the neoplastic cells.

Despite the malignancies features observed in the histopathological evaluation of the myoepithelioma in this case report, no infiltration of neoplastic cells into lymphatic vessels or lymph nodes were observed which may indicate that metastasis in these animals may occur as a late event in the progression of the disease; since in dogs the metatastic potential of this histological type is low [17]. It is important to note that due to the difficulty on the identification and resection of the axillary lymph node, and absence of validated studies with vital stains in lagomorphs, the excision and histological evaluation of the axillary lymph node were not performed. No complementation was made to the surgical treatment, due to the lack of evidence in the literature, however, despite the reserved prognosis, a disease-free interval of more than 405 days was observed, without abnormalities in the complementary and staging exams.
Rabbits present three to five pairs of mammary glands named cranial and caudal thoracic, cranial and caudal abdominal, and inguinal [5], in this case, the patient had three pairs of mammary glands (M1-3). Mammary neoplasms in rabbits are common in intact females over 3 to 4 years but have been reported from 2 to 14 years of age with an average of 5.5 years [3], consistent with the rabbit's age of this report. Some of these cases have been also associated with prolactin-producing pituitary adenomas or uterine hyperplasia and uterine adenocarcinomas [3,4,8,21]. In two studies $[16,22]$, both in nulliparous rabbits, pituitary adenoma was assigned as a predisposing factor to cystic mammary adenocarcinoma, dysplasia and hyperplasia. The pituitary adenoma secretes prolactin, which results in dysplastic abnormalities in the mammary glands, which are overresponsive to prolactin in rabbits if compared to other mammals [16]. In this case, it was not possible to associate it with pituitary neoplasia since it would require brain evaluation by computed tomography or magnetic ressonance imaging. However, the animal in the present case showed no evidence of other hormonal abnormalities, such as hyperadrenocorticism, which commonly occur in cases of pituitary neoplasia.

In females, after puberty, several ovarian follicles and luteal bodies occupy the ovarian parenchyma, and a strong influence of oestrogen and progesterone on the uterine tissue is recognized on these animals, which may favor endometrial hyperplasia and neoplasia [1]. Tumour development seems to be often preceded by the development of cystic abnormalities in the mammary gland [11]. In a study with a colony of breeding rabbits, most of the mammary tumours developed from cystic mammary disease, postulating a progression to benign neoplasia followed by invasive carcinomas [20]. In their study of mammary gland in rabbits, 10 animals (42\%) simultaneously had several tumour lesions (lobular hyperplasia, cysts and neoplasms) suggesting a predisposition of domestic rabbits for the development of multiple mammary lesions, which often occurs in bitches $[9,20]$. It was demonstrated in this report that the rabbit presented one malignant neoplasm and two cystic lesions, ipsilaterally.

The pseudocyesis occurs due to the presence of a male or when it is mounted by another female [5], followed by ovulation but not fertilization. It may last 15 to 17 days, due to luteal formation, which persists for 18 to 21 days increasing progesterone 
secretion, that leads to mammary enlargement along with uterine hyperplasia [26]. Reproductive diseases such as uterine or mammary neoplasm and endometritis often occur in middle aged rabbits, while endometrial hyperplasia has been diagnosed in rabbits over one year old $[12,26]$. According to some authors $[1,25,26]$, under hormonal influence, cystic endometrial hyperplasia may predispose to uterine adenocarcinoma, and mammary gland pathologies $[3,10]$. In one study, with 47 rabbits with uterine lesions, $31.9 \%$ presented mammary cysts identified by physical examination [19]. Two studies reported a high incidence of cystic mammary lesions in rabbits with endometrial hyperplasia or uterine adenocarcinoma, but mammary carcinomas were associated only with uterine adenocarcinomas [12,26]. However, Baum and Hewicker-Trautwein [3] found uterine diseases only in three of the 89 rabbits with benign and malignant neoplasms of the mammary gland. The patient of this study presented ultrasonographical abnormalities, compatible with uterine hyperplasia, which could evolve into a uterine adenocarcinoma as seen in other studies [20]. Nevertheless it is more likely that all this conditions are only partially related to oestrogen and oprogesterone levels.

As in dogs and cats, neutering is also indicated for rabbits in the treatment of neoplasic and non-neoplasic uterine lesions [12,26], which can be performed at any age, although Harcourt-Brown [11] advises the sterilization of males and females older than five months. In the case reported, considering the likelihood of malignant mammary disease, suggested by cytology, neutering was not indicated concomitantly to the radical mastectomy, since it had been proven that the association of such procedures in dogs enhances the organic response to surgical trauma and may promote dissemination of the neoplasia [18]. However, after six months of follow-up, and based on the ultrasonographical abnormalities, and aiming at the prevention of complications, the ovary-hysterectomy was indicated, although rejected by the pet's responsible.

The rabbit of the present report had a histological diagnosis of a malignant myoepithelioma, according to the WHO classification, and confirmed by the immunohistochemistry with the positive immunolabeling for the myoepithelial p63 and negative for the epithelial and mesenchymal cell markers. This is the first report of this type of mammary neoplasia in rabbits. The neoplasia and breast cysts were removed by mastectomy, and the pet's responsible choose not to spay the rabbit. Abdominal ultrasound revealed endometrial hyperplasia along with cystic lesions, probable related to hormone balance (oestrogen and progesterone), but they did not show signs of progression during clinical follow-up.

\section{MANUFACTURERS}

${ }^{1}$ Vetnil Industria e Comércio Ltda. Louveira, SP, Brazil.

${ }^{2}$ Syntec do Brasil Ltda. Santana de Parnaíba, SP, Brazil.

${ }^{3}$ Hipolabor Farmacêutica Ltda. Belo Horizonte, MG, Brazil.

${ }^{4}$ Laboratório Cristália. São Paulo, SP, Brazil.

${ }^{5}$ Dako North America Inc. Carpinteria, CA, USA.

${ }^{6}$ Neomarkers. Freemount, CA, USA.

Declaration of interest. The authors report no conflicts of interest. The authors alone are responsible for the content and writing of the paper.

\section{REFERENCES}

1 Asakawa M.G., Goldschmidt M.H., Une Y. \& Nomura Y. 2008. The immunohistochemical evaluation of estrogen receptor- $\alpha$ and progesterone receptors of normal, hyperplastic, and neoplastic endometrium in 88 pet rabbits. Veterinary Pathology. 45(2): 217-225.

2 Bauchet A.L., Elies L., Maliver P., Fouque M.C., Balme E., Château-Joubert S., Schorsch F. \& Fontaine J.J. 2008. A mammary gland adenomyoepithelioma in a C57BL/6 mouse. Experimental and Toxicologic Pathology. 60(45): 307-311.

3 Baum B. \& Hewicker-Trautwein M. 2015. Classification and Epidemiology of Mammary Tumors in Pet Rabbits (Oryctolagus cuniculus). Journal of Comparative Pathology. 152(4): 291-298.

4 Cassali G.D., Lavelle G.E., De Nardi A.B., Ferreira E., Bertagnolli A.C., Estrela-Lima A., De Nardi A.B., Ghever C., Sobral R.A., Amorim R.L., Oliveira L.O., Sueiro F.A.R., Beserra H.E.O., Bertagnolli A.C., Gamba C.O., Damasceno K.A., Campos C.B., Araujo M.R., Campos L.C., Monteiro L.N., Nunes F.C., Horta R.S., Reis D.C., Luvizotto M.C.R., Magalhães G.M., Raposo J.B., Ferreira A.M.R., Tanaka N.M., Grandi F., Ubukata R., Batschinski K., Terra E.M., Salvador R.C.L., Jark P.C., Delecrodi J.E.R., Nascimento N.A., Silva D.N., Silva 
L.P., Ferreira K.C.R.S., Frehse M.S., Santis G.W., Silva E.O., Guim T.N., Kerr B., Cintra P.P., Silva F.B.F., Leite J.S., Mello M.F.V., Ferreira M.L.G., Fukumasu H., Salgado B.S. \& Torres R. 2014. Consensus for the diagnosis, prognosis and treatment of canine mammary tumours. Brazilian Journal Veterinary Pathology. 7(2): 38-69.

5 Couto S.E.R. 2002. Criação e manejo de coelhos. In: Andrade A., Pinto S.C. \& Oliveira R.S. (Eds). Animais de Laboratório: criação e experimentação [online]. Rio de Janeiro: FIOCRUZ, pp.98-99.

6 Faustino A.M. \& Pereira P.D. 2007. A salivary malignant myoephitelioma in a dog. Veterinary Journal. 173: 223-226.

7 Gama A., Alves A., Gartner F. \& Schimit F. 2003. p63: A Novel Myoepithelial Cell Marker in Canine Mammary Tissues. Veterinary Pathology. 40(4): 412-420.

8 Goldschmidt M., Penã L., Rasotto R. \& Zappulli V. 2011. Classification and Grading of Canine Mammary Tumors. Veterinary Pathology. 48(1): 117-131.

9 Greene H.S.N. \& Strauss J.S. 1949. Multiple primary tumors in the rabbit. Cancer. 2(4): 673-691.

10 Greene H.S.N. 1939. Familial mammary tumors in the rabbit: I. clinical history, II. Gross and microscopic pathology. Journal of Experimental Medicine. 70(2): 147-166.

11 Harcourt-Brown F. 2002. Urogenital diseases. In: Varga M. (Ed). Textbook of Rabbit Medicine. Woburn: Reed Educational and Professional Publishing, pp.350-351.

12 Künzel F., Grinninger P., Shibly S., Hassan J., Tichy A., Berghold P. \& Fuchs-Baumgartinger A. 2015. Uterine disorders in 50 pet rabbits. Journal of the American Animal Hospital Association. 51(1): 8-14.

13 Lakhani S.R., O'Hare M.J., Monaghan P., Winehouse J., Gazet J.C. \& Sloane J.P. 1995. Malignant myoepithelioma (Myoepithelioma carcinoma) of the breast: a detailed cytokeratin study. Journal of Clinical Pathology. 48(2): 164-167.

14 Liang Y., Zeng J., Kang D., Wang L., Chen C., Xu J. \& Wu Q. 2014. Malignant myoepithelioma of the breast: a case report and review of literature. International Journal of Clinical and Experimental Pathology. 7(5): 2654-2657.

15 Papazian M., Kalantzis I., Galanopoulos G., Mani I., Tzaida O., Iacovidou I. \& Ziras N. 2016. Malignant myoepithelioma of the breast: A case report and review of the literature. Molecular and Clinical Oncology. 4(5): 723-727.

16 Percy D.H. \& Barthold S.W. 2007. Rabbit. In: Pathology of Laboratory Rodents and Rabbits. 3rd edn. Ames: Blackwell, pp.310-313.

17 Rasotto R., Zappulli V., Castagnaro M. \& Goldschmidt M.H. 2012. A Retrospective Study of Those Histopathologic Parameters Predictive of Invasion of the Lymphatic System by Canine Mammary Carcinomas. Veterinary Pathology. 49(2): 330-340.

18 Rodigheri S.M. 2016. Parâmetros da resposta metabólica ao trauma cirúrgico induzido por mastectomia unilateral associada ou não à ovariohisterectomia em cadelas. 82f. Jaboticabal, São Paulo. Tese (Doutorado em Cirurgia Veterinária) - Programa de Pós-graduação em Cirurgia Veterinária, Universidade Estadual Paulista.

19 Saito K., Nakanishi M. \& Hassegawa A. 2002. Uterine disorders diagnosed by ventrotomy in 47 rabbits. Journal of Veterinary Medical Science. 64(6): 495-497.

20 Schöniger S., Horn L.C. \& Schoon H.A. 2014. Tumors and tumor-like lesions in the mammary gland of 24 pet rabbits: a histomorphological and immunohistochemical characterization. Veterinary Pathology. 51(3): 569-80.

21 Shahbazfar A.A., Mohammadpour H. \& Isfahani H.R. 2012. Mammary Gland Carcinosarcoma in a New Zealand White Rabbit (Oryctolagus cuniculus). Acta Scientiae Veterinariae. 40(1): 1025.

22 Sikoski P., Trybus J., Cline J.M., Muhammad F.S., Eckhoff A., Tan J., Lockard M., Jolley T., Britt S. \& Kock N.D. 2008. Cystic mammary adenocarcinoma associated with a prolactin-secreting pituitary adenoma in a New Zealand White rabbit (Oryctolagus cuniculus). Comparative Medicine. 58(3): 297-300.

23 Tan P.H. \& Ellis I.O. 2013. Myoepithelial and epithelial-myoepithelial, mesenchymal and fibroepithelial breast lesions: updates from the WHO Classification of Tumors of the Breast 2012. Journal of Clinical Pathology. 66(6): 465-470.

24 Terada T. 2011. Malignant myoepithelioma of the breast. Pathology International. 61(2): 99-103.

25 Vinci A., Bacci B., Benazzi C., Caldin M. \& Sarli G. 2010. Progesterone Receptor Expression and Proliferative Activity in Uterine Tumours of Pet Rabbits. Journal of Comparative Pathology. 142(4): 323-327.

26 Walter B., Poth T., Bohmer E., Braun J. \& Matis U. 2010. Uterine disorders in 59 rabbits. Veterinary Record. 166(8): 230-233. 